\title{
Discutendo di guerra
}

Il centenario della Prima guerra mondiale costringe gli studiosi e le opinioni pubbliche a riflettere ancora una volta attorno ai caratteri e alle conseguenze di quell'enorme fenomeno storico.

Atto di nascita del secolo breve ${ }^{1}$, momento di svolta di una modernità più lunga ${ }^{2}$, avvio della guerra dei trent'anni ${ }^{3}$, origine della memoria moderna ${ }^{4}$, prova globale : comunque gli storici l'abbiano qualificato, in termine di drammatica cesura o di decisivo tornante, è difficile trovare un altro momento della storia contemporanea che abbia attratto e attragga discussioni più durature, che appunto oggi compiono un secolo. Sono discussioni che parlano di guerra e di pace, di morte di massa e di sospensione di diritti, di rivoluzione e di soggettività, e che cadono oggi, dovunque, mentre numerosi conflitti armati continuano ad insanguinare il pianeta e mentre la pace, i diritti, le soggettività sono di nuovo sotto attacco. Difficile quindi attendersi discussioni storiografiche pacifiche e ireniche.

Ci sono stati paesi in cui allora si stabilì (e oggi, pur trasformato, si ripresenta) un qual certo consenso nazionale su alcuni punti di fondo della parte-

Come Eric J. Hobsbawm, Il secolo breve (1994), Milano, Rizzoli, 1995.

${ }^{2}$ Nelle due versioni di Arno J. Mayer, Il potere dell'ancien régime fino alla Prima guerra mondiale (1981), Roma-Bari, Laterza, 1982, e Charles S. Maier, Secolo corto o epoca lunga? L'unità storica dell'età industriale e le trasformazioni della territorialità, in Claudio Pavone (a cura di), Novecento. I tempi della storia, Roma, Donzelli, 1997, pp. 45-78.

${ }^{3}$ Un taglio ripreso da Ernst Nolte, Nazionalsocialismo e bolscevismo: la guerra civile europea, 1917-1945, con un saggio di Gian Enrico Rusconi, Firenze, Sansoni, 1989.

${ }^{4}$ È la nota tesi di Paul Fussell, La Grande guerra e la memoria moderna (1975), Bologna, Il Mulino, 1984.

${ }^{5}$ Per una lucida indicazione si veda Daniel Marc Segesser, Der erste Weltkrieg in globaler Perspektive, Wiesbaden, Marix Verlag, 2010.

"Italia contemporanea", aprile 2016, n. 280 
cipazione alla Prima guerra mondiale ${ }^{6}$ E anche in Italia ${ }^{7}$ il processo di nazionalizzazione fece passi avanti, sia pur - appunto - nel sangue della guerra. La stessa vittoria finale, niente affatto scontata e anzi messa a duro rischio nel maggio 1916, nell'ottobre-novembre 1917 e sino al giugno 1918, vi contribuì. Ma la spaccatura dello stesso fronte liberale al momento dell'entrata in guerra, lo sforzo enorme subito dal paese, la mancanza di governi di "unione sacra", la presenza dell'unico grande Partito socialista europeo che non votò i crediti di guerra, non erano segni di unificazione bensì di divisione. Inoltre, quanto avvenne dopo la fine del conflitto, fra dopoguerra, crisi finale del sistema liberale ed avvento del fascismo, sia pure non addebitabile meccanicisticamente alla Grande guerra, nemmeno può essere ignorato. Per tutte queste ragioni, ancora oggi discutere del primo conflitto mondiale - peraltro, come osservavamo, in tempi di guerra - difficilmente può essere considerato un esercizio accademico.

D'altro canto, che la Grande guerra conservi da noi un tale carattere di discussione, e non di consenso, potrebbe essere visto con soddisfazione, e senza esecrazioni di un presupposto carattere divisivo della storiografia naziona$l^{8}$, non foss'altro nella misura in cui la discussione e la ricerca possono servire da stimolo a chiarificare aspetti ancora poco noti, o ad affinare metodi d'indagine poco praticati, o a identificare questioni e fonti storiche ancora trascurate.

Quando si leggeranno queste righe, il centenario sarà in Europa ormai a circa un terzo del suo cammino. Era stato avviato dai più previdenti e preparati, autori o editori, quantomeno dal $1913^{9}$. È formalmente partito di fatto dovunque (anche in Italia) nel 1914 sia con la discussione di volumi che hanno fatto (e sembravano fatti per apposta per) discutere ${ }^{10}$, sia soprattutto con l'edizione di grandi ricostruzioni dal taglio generale ${ }^{11}$ e di opere collettive internazionali e transnazionali: dalla Cambridge History of the First World War ${ }^{12}$ alla

${ }^{6} \mathrm{Si}$ veda Antoine Prost, Jay Winter, Penser la Grande Guerre. Un essai d'historiographie, Paris, Seuil, 2004.

${ }^{7}$ Si pensa a Mario Isnenghi, Giorgio Rochat, La Grande guerra 1914-1918, Firenze, La Nuova Italia, 2000 (nella collana Storia d'Italia nel secolo ventesimo, promossa dall'Istituto nazionale per la storia del movimento di liberazione in Italia), riedita da Sansoni, e poi più volte da Il Mulino; Antonio Gibelli, La Grande guerra degli italiani 1915-1918, Milano, Sansoni, 1998; e Giovanna Procacci, La Prima guerra mondiale, in Giuseppe Sabbatucci, Vittorio Vidotto (a cura di), Storia d'Italia, vol. IV, Guerre e fascismo, Roma-Bari, Laterza, 1997.

${ }^{8} 24$ maggio 1915 l'Italia è in guerra, Milano, Corriere della Sera, 2015.

${ }^{9}$ Florian Illies, 1913 l'anno prima della tempesta, Venezia, Marsilio, 2013.

${ }^{10}$ Christopher Clark, I sonnambuli. Come l'Europa arrivò alla Grande guerra, Roma-Bari, Laterza, 2013.

${ }^{11} \mathrm{Nel}$ panorama editoriale italiano, ricordo innanzitutto la buona sintesi di Oliver Janz, 19141918 la grande guerra (2013), Torino, Einaudi, 2014, purtroppo funestata da una traduzione inesperta su questi temi.

${ }^{12}$ Jay Winter (a cura di), The Cambridge History of the First World War, Cambridge, Cambridge University Press, 2014. 
enciclopedia open access 1914-1918 on line li $^{13}$. In quello stesso 1914 la rilevanza dell'evento centenario è stata accompagnata, nei vari paesi, da solenni manifestazioni (che andranno in un futuro studiate) e con la costituzione, l'attività e il rilevante finanziamento (non in Italia) di strutture e comitati nazionali per le commemorazioni. A livello internazionale ha poi marciato con buon passo nel 1915, sia pure in genere producendo opere di minore impatto.

In Italia, ovviamente, in questo 1915 appena concluso, il centenario si è fatto sentire maggiormente. Lo hanno contrassegnato alcune, poche, iniziative nazionali di grande eco ${ }^{14}$, alcuni volumi di sintesi o di taglio innovativo, una miriade di iniziative disperse nei diversi comuni d'Italia. Vi è stato anche un bando pubblico di finanziamento, che ha sollecitato quasi novecento enti o associazioni a fare domanda: una lunga lista, dal quale sono state estratte, a seguito di un primo vaglio di un comitato di storici - ma alla fine con modalità non del tutto chiarite pubblicamente - quarantacinque iniziative ${ }^{15}$. Una buona attenzione dei mezzi di comunicazione ha assicurato una certa rilevanza a molte attività.

Finora, in questo centenario italiano, ha prevalso la continuità o la discontinuità? la ripetizione o l'innovazione? la quantità o la qualità? In generale, è noto, le commemorazioni e le ricorrenze non sempre offrono l'occasione migliore per un ripensamento e un rinnovamento: le celebrazioni raramente sono momenti di formazione di un pensiero critico in grado di lasciarsi alle spalle la retorica. Certo all'interno di una mole così imponente di studi (il Servizio bibliotecario nazionale censisce almeno quattrocento pubblicazioni, edizioni o riedizioni, di monografie, grandi o piccole, stampate fra il 2014 e il 2015 sui temi della Prima guerra mondiale: e questo senza contare gli articoli delle riviste scientifiche) non poteva non esserci stata anche qualità. Ma in quale direzione si è andati? In quale si sta andando? Quale traiettoria abbozzano questi avvii rispetto ai migliori studi internazionali?

Fra i tanti titoli pubblicati non abbiamo per adesso avuto nuove storie nazionali e generali: la maggiore rimane quindi ancora quella scritta da Mario Isnenghi e Giorgio Rochat ${ }^{16}$, più volte ristampata, affiancata da quella - di poco precedente - di Antonio Gibelli ${ }^{17}$ e dalla più veloce traccia di Giovanna Procacci ${ }^{18}$. Segno dei tempi, abbiamo invece assistito ad una rivalorizzazione dell'opera, del 1969 e da allora mai aggiornata, di Piero Melograni ${ }^{19}$. Abbiamo

${ }^{13} \mathrm{Si}$ veda http://www.1914-1918-online.net/.

${ }^{14} \mathrm{http} / / / \mathrm{www} . c e n t e n a r i o 1914-1918$.it/it.

15 http://www.centenario1914-1918.it/it/2015/11/18/graduatoria-delle-iniziative-culturali-bando-grande-guerra.

${ }^{16}$ Mario Isnenghi, Giorgio Rochat, La Grande guerra 1914-1918, cit.

${ }^{17}$ Antonio Gibelli, La Grande guerra degli italiani 1915-1918, cit.

${ }^{18}$ Giovanna Procacci, La Prima guerra mondiale, cit.

${ }^{19}$ Piero Melograni, Storia politica della Grande guerra 1915-1918, Bari, Laterza, 1969, ried. da Milano, Mondadori, 2014. 
avuto però alcune messe a punto generali, in forma collettiva e sintetica ${ }^{20} \mathrm{o}$ individuale e analitica ${ }^{21}$.

Maggiori novità è stato possibile trovare in alcune opere dal taglio loca$\mathrm{le}^{22}$ o regionale, una dimensione nuova degli studi sulla Grande guerra, particolarmente utile quando sotto la lente degli studiosi sono passati i territori meridionali, meno conosciuti per quel periodo 1914-1918. Un'attenzione nuova, per adesso più mediatica e politica che storiografica, è stata portata ad alcune categorie di "vittime" della Grande guerra italiana: soprattutto, i fucilati ${ }^{23}$. Una nuova serie di studi e di pubblicazioni è stata rivolta al ruolo delle donne ${ }^{24}$ (per la verità, soprattutto, ancora, borghesi e cittadine), uno spazio di ricerca amplissimo ancora in buona parte da sondare. Una ricerca collettiva ha infine cercato di fare maggiore luce sull'insediamento territoriale dei neutralisti ${ }^{25}$, principalmente liberali. Insomma, non sono mancati temi nuovi o il cui studio è stato rinnovato.

Altri temi, pur importanti, hanno ricevuto nuova attenzione, ma trattazioni più tradizionali, meno innovative. Si badi bene: pur qualificate e qualificanti la storiografia italiana, sono state narrazioni tutto sommato già note. Alcuni esempi: le vite degli umili travolti dalla guerra ${ }^{26}$, i soldati e la loro storia intima fra consenso e dissenso ${ }^{27}$, soprattutto molta storia politi-

${ }^{20}$ Sia concesso di rinviare a Nicola Labanca, Oswald Überegger (a cura di), La guerra italo-austriaca 1915-18, Bologna, Il Mulino, 2014 (anche in ediz. austriaca Krieg in den Alpen. Österreich-Ungarn und Italien im Ersten Weltkrieg (1914-1918), Wien-Köln-Weimar, Böhlau, 2015), e a Nicola Labanca (a cura di), Dizionario storico della Prima guerra mondiale, RomaBari, Laterza, 2014.

${ }^{21} \mathrm{Si}$ veda l'ampio e ambizioso Marco Mondini, La guerra italiana: partire, raccontare, tornare 1914-18, Bologna, Il Mulino, 2014, il cui vero senso si intende in Id., L'historiographie italienne face à la Grande Guerre: saisons et ruptures, "Histoire@Politique. Politique, culture, société", 2014, n. 22, liberamente consultabile in www.histoire-politique.fr.

${ }^{22} \mathrm{Si}$ veda per esempio Giuseppe Barone (a cura di), Catania e la Grande guerra. Storia, protagonisti, rappresentazioni, Acireale, Bonanno, 2014.

${ }^{23} \mathrm{Si}$ veda, dopo una serie di interventi sul quotidiano "Avvenire" nell'estate 2014 curati da Giovanni Grasso, il convegno tenutosi a Rovereto nel maggio 2015, su cui http://www.museodellaguerra.it/convegno-litalia-nella-guerra-mondiale-e-i-suoi-fucilati-quello-che-non-sappiamo-2/.

${ }^{24}$ Fra i molti si vedano Donne nella grande guerra, introduzione di Dacia Maraini, Bologna, Il Mulino, 2014; Augusta Molinari, Una patria per le donne. La mobilitazione femminile nella grande guerra, Bologna, Il Mulino, 2014; Daniela Rossini, Donne e propaganda internazionale. Percorsi femminili tra Italia e Stati Uniti nell'età della Grande guerra, Milano, FrancoAngeli, 2015.

${ }^{25} \mathrm{Si}$ veda Fulvio Cammarano (a cura di), Abbasso la guerra! Neutralisti in piazza alla vigilia della Prima guerra mondiale in Italia, Firenze, Le Monnier università-Mondadori education, 2015.

${ }^{26}$ Antonio Gibelli, La guerra grande. Storie di gente comune 1914-1919, Roma-Bari, Laterza, 2014. Dello stesso autore si veda anche la recente riedizione di alcuni dei suoi molti interventi sul tema sotto il titolo di Il colpo di tuono. Pensare la Grande guerra oggi, Roma, Manifestolibri, 2015.

${ }^{27}$ Si pensa a Quinto Antonelli, Storia intima della Grande guerra. Lettere, diari e memorie dei soldati dal fronte, con un Dvd del film di Enrico Verra Scemi di guerra, Roma, Donzelli, 2014. 
$\mathrm{ca}^{28}$. Si è trattato di ottime ricostruzioni o tratteggiamenti, a firma di specialisti riconosciuti del settore, che pure probabilmente andavano incontro al favore del pubblico: ma i cui metodi e i cui risultati non hanno stupito gli specialisti.

Dove la bilancia ha naturalmente segnato la prevalenza del noto sulla sorpresa è stato nelle opere di giornalisti e pubblicisti, i quali non si sono fatti sfuggire l'occasione del centenario per confezionare - con provata esperienza - opere che, a differenza di quelle degli storici, sembravano fatte per andare incontro al favore del pubblico. $\mathrm{O}$ meglio dei pubblici: così il pubblico più generale, quello più riflessivo e quello più impegnato hanno avuto ognuno le proprie letture di riferimento sulla Grande guerra, per esempio, per la penna rispettivamente di Aldo Cazzullo ${ }^{29}$, Paolo Rumiz ${ }^{30}$ e del collettivo Wu Ming ${ }^{31}$. Opere tutte diverse fra loro, quindi, sia pure - a pensarci bene - accomunate da un richiamo al "popolo", e talora ad un certo populismo, evidente segno dei tempi. La stessa recentissima riedizione del Diario di guerra di Benito Mussolini (1916) per conto di ben tre case editrici e per la diversa curatela di tre studiosi di ben diverso orientamento (Mario Isnenghi ${ }^{32}$, Alessandro Cam$\mathrm{pi}^{33}$ e Mimmo Franzinelli ${ }^{34}$ ) lascia intendere di come si sia pensato che a ogni pubblico si sia voluto vendere la "propria" Grande guerra. Andare contro operazioni culturali così pensate e sfidare le corazzate editoriali dei libri di queste "grandi firme" del mondo della comunicazione nazionale è stato per gli storici assai difficile ed è probabile che acute messe a punto o reinterpretazioni di fini ed esperti storici - pensiamo in particolare al raffinato Convertirsi alla guer$r a^{35}$ di Mario Isnenghi - o nuove offerte a firma di più giovani ma già ben caratterizzati studiosi - pensiamo a Marco Mondini e al suo Andare per luoghi

${ }^{28}$ Un esempio: Francesco Perfetti (a cura di), Niente fu più come prima. La Grande guerra e l'Italia cento anni dopo. Atti del Convegno, Firenze, 13-14 marzo 2015, Firenze, Polistampa, 2015.

${ }^{29}$ Aldo Cazzullo, La guerra dei nostri nonni 1915-1918. Storie di uomini, donne, famiglie, Milano, Mondadori, 2015.

${ }^{30}$ Si pensa qui al passaggio dalla commemorazione alla rievocazione di Paolo Rumiz, Come cavalli che dormono in piedi, Milano, Feltrinelli, 2014.

${ }^{31} \mathrm{Si}$ vedano Wu Ming 1, Cent'anni a Nordest. Viaggio tra i fantasmi della guera granda, Milano, Rizzoli, 2015; Wu Ming, L'invisibile ovunque, Torino, Einaudi, 2015; e Wu Ming, L'armata dei sonnambuli, Torino, Einaudi, 2014.

32 Benito Mussolini, Il mio diario di guerra, a cura di Mario Isnenghi, Bologna, Il Mulino, 2016.

${ }^{33}$ Benito Mussolini, Giornale di guerra 1915-1917, a cura di Alessandro Campi, Soveria Mannelli, Rubbettino, 2016.

${ }^{34}$ Benito Mussolini, Giornale di guerra, 1915-1917, a cura di Mimmo Franzinelli, Gorizia, Goriziana, 2016. Per la precisione si era già registrata di recente un'iniziativa locale, la riedizione dello stesso volume a cura di Denis Vidale, Castelfranco Veneto, Biblioteca dei leoni, 2015, e una inquietante presso Padova, Edizioni di Ar, 2015.

${ }_{35}$ Mario Isnenghi, Convertirsi alla guerra. Liquidazioni, mobilitazioni e abiure nell'Italia tra il 1914 e il 1918, Roma, Donzelli, 2015. 
della Grande guerra ${ }^{36}$ - si siano trovate in termini di copie vendute a grande distanza.

Altre occasioni e altri momenti saranno necessari per redigere un accurato bilancio che - scendendo nei dettagli e nell'esame accurato delle pubblicazioni anche minori presso le più appartate sedi editoriali — potrà portare forse a conclusioni più ottimistiche. Certo la prima impressione è che, per adesso, la continuità abbia fatto aggio sulla discontinuità, la rielaborazione sulla sorprendente innovazione, o sulla reinterpretazione ${ }^{37}$.

Continua in particolare un certo divario, e non è un buon segno, con le storiografie di altri paesi: sono del tutto mancate fra gli studiosi italiani le forze e le volontà per qualche grande storia internazionale e complessiva della Grande guerra ${ }^{38}$, affreschi che invece altrove si sono moltiplicati, mettendo in evidenza i tratti più innovativi delle interpretazioni più aggiornate, che insistono su un conflitto visto come una guerra globale, totale, profondamente segnata da fenomeni transnazionali. Colpisce ancora di più una certa distanza degli interessi delle storiche e degli storici italiani da molti fra i più fertili campi di ricerca quali opere come i tre tomi della Cambridge History of the First World War hanno configurato: non tanto per quanto attiene al primo volume (se vogliamo, il più tradizionale) dedicato alle caratteristiche militari e politiche della guerra, ma per quanto è là trattato nel secondo volume sull'azione dello Stato (ancora un grande assente, negli studi italiani, a parte un recente convegno) e soprattutto nel suo terzo e davvero innovativo volume, sulla storia sociale e culturale-sociale $^{39}$. Su quest'ultimo punto, davvero, la storiografia italiana è complessivamente piuttosto assente, o carente, nonostante si tratti di temi forse non esplicitati ma certo già quanto meno accennati da un'altra grande opera, di quasi dieci anni fa, pure meritoriamente tradotta in italiano ${ }^{40}$. In una parola, a un primissimo sguardo, gli studi italiani sembrano non aver colmato del tutto certi divari formatisi negli ultimi decenni con la storiografia internazionale.

Ma forse si tratta di impressioni, e comunque il centenario è tutt'altro che finito.

"Italia contemporanea" - anche per inaugurare degnamente la nuova sezione "In rete" — ha colto l'occasione per avviare una riflessione storiografica su tutto questo grazie alla disponibilità di due fra i più noti studiosi italiani della

${ }^{36}$ Marco Mondini, Andare per i luoghi della Grande guerra, Bologna, Il Mulino, 2015.

${ }^{37}$ Un'eccezione forse Diego Leoni, La guerra verticale. Uomini, animali e macchine sul fronte di montagna, 1915-1918, Torino, Einaudi, 2015.

${ }^{38}$ L'unica eccezione è la riedizione della Piccola storia della Grande guerra di Angelo Ventrone (Roma, Donzelli, 2005) come Id., Grande guerra e Novecento. La storia che ha cambiato il mondo, Roma, Donzelli, 2015.

${ }^{39}$ Jay Winter (a cura di), The Cambridge History of the First World War, cit.

${ }^{40} \mathrm{Si}$ veda Stéphane Audoin-Rouzeau, Jean-Jacques Becker (a cura di), La Prima guerra mondiale, edizione italiana a cura di Antonio Gibelli, Torino, Einaudi, 2007, di recente riedita in edizione tascabile in brossura. 
Prima guerra mondiale come Mario Isnenghi e Giovanna Procacci. Due studiosi che nelle pagine che seguono parlano dello stato e dell'indirizzo degli studi all'altezza del centenario, ma anche di sé e del proprio percorso di formazione, e parlano l'una dell'altro, nella consapevolezza che di discutere della Grande guerra è discutere della storia d'Italia. Non parlano di tutto, ovviamente, ma di molto: con la convinzione che discutere di guerra solleva sempre grandi implicazioni.

n.l. 